\title{
A web-based test system using ASP.NET and AJAX
}

\author{
Li Yingxia \\ Hubei Urban Construction Vocational and Technical \\ College \\ Wuhan, China \\ $5001360 @ q q . c o m$
}

\author{
Hao Zimian \\ Hubei Urban Construction Vocational and Technical \\ College \\ Wuhan, China \\ haozimian@whu.edu.cn
}

\begin{abstract}
Aiming at the low efficiency of the traditional examination, waste of human and material resource, this paper presents the design and implementation of a webbased test system based on $B / S$ structure. The system realizes efficient, flexible arrangement of multi subject test, achieves low cost to organize students examination, automatic scoring, paper archive, and is convenient for students to read the history record, Checks the scores and knowledge of each test questions, at the same time, while using the backend system calling Microsoft office components, to achieve a key generation related documents function. After many hundreds people at the same time tests prove that the system is stable and efficient.
\end{abstract}

Keywords- Microsoft office Component; ASP.NET; AJAX; test system; WEB application

\section{INTRODUCTION}

With the rapid development of Internet and computer technology, modern education based on the Internet as the main means of today has become the trend of education development. Examination is an important part of education process, it can not only arouse the learning enthusiasm of students, also can let the teacher know students to master the knowledge, do have a definite object in view, and improve classroom efficiency [1]. In the traditional test way, organize an examination is a multistage process: teachers, printed papers, tissue examination of student, teacher, the statistical results and evaluation and examination paper analysis, consumes a lot of manpower and material resources, and in the papers, the work is also very prone to human error. But, as the number of examination questions and the examinee continues to increase and improve the exam, the organization of the examination of the increasingly heavy workload, but also more and more complicated. As Internet popularize vigorously, in order to save cost, ensure the fairness of the exam science, improve the efficiency, the paper test onto the network online examination mode has become represent the general trend[2,3].

The system breaks the traditional examination method, not only can flexibly arrange the test, Objectively and accurately evaluate people in a certain ability and level in the field of knowledge, improve test efficiency greatly, Let the examination work simplification, modeled, transparent, but also convenient for students to read the history examination, score and check each question, so that the more solid grasp of knowledge. Compared with the traditional examination, it can use the infinite space network whenever and wherever possible, let the students practice, testing and examination, coupled with the use of database technology, greatly simplifies the traditional examination process, improve the quality of examination and evaluation.

\section{RELATED TECHNOLOGY}

\section{A. ASP.NET Technology}

ASP.NET is not only the next edition of Active Server Page (ASP), it creates a unified model for development of Web-based applications, including a variety of services to enable a programmer to use as little as possible code generation enterprise level necessary for Web applications. ASP.net provides much powerful advantage [4].

1) Execution efficiency: ASP.net runs the programs Based on the common language on the server-side. When A page is requested for the first time, it will be compiled by common language runtime (CLR) compiler. When this page is accessed again, because it has been compiled, The common language runtime directly execute the compiled code, which is compiled once executed many times, can greatly improve the efficiency of execution.

2) Strong and adaptability: Because ASP.net is based on the common language compiler running program, so the the base Library, message mechanism, data interface of the common language can be seamlessly integrated into the Web application in ASP.net.

3) Manageability:ASP.net using a hierarchical configuration system, configuration information is stored in a simple text file, the new settings can be very simple and quick[5]. When it wants to install the ASP.net application on the server, it just need to copy the necessary files.

4) Security:Based on Windows authentication technology and application configuration, the ASP.net source program is absolutely safe. 


\section{B. ADO.NET Technology}

In the process of WEB development, access to the database is a very important link, especially in the $\mathrm{B} / \mathrm{S}$ construction system, database operation is almost an indispensable operation. ADO.NET is the classes set of .NET Framework which specific to operate on the data in the database. It is an important technology for data exchange between WEB pages and database. Is an important bridge between front-end and backend database.

ADO.NET provides uniform access to various data sources, can use ado.net to connect to the data source, and retrieval, processing and updating the data, ado.net can separate data access from data processing. Using ADO.NET, user can develop application of database related fast and efficiently.The ADO.NET structure is shown as "Fig. 1".

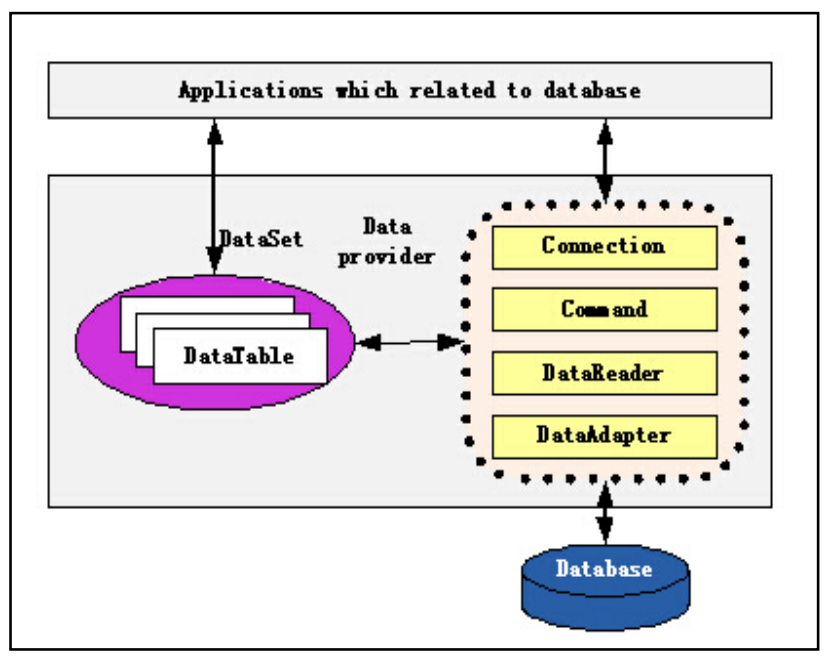

Figure 1. ADO.NET structure.

\section{AJAX technology}

Asynchronous JavaScript + XML hereinafter referred to as AJAX, it is combined with the existing technology, including the cascading style sheets, JavaScript, XHTML, $\mathrm{XML}$ and extensible style sheet language transformation, and the synthesis of a powerful new technology. AJAX is a WEB application development method to use the client script realizing web server interactive data. Using Ajax's asynchronous mode, the browser without user request operation, also don't have to update the entire window can display new acquired data. Using data in XML format, sending messages between the client and server, by running the JavaScript code, it can contact web server in browser. JavaScript code can also add the stylesheet to retrieve the data, and then show it in some part of the existing web page. Through this type, it can avoids browser page reloading that user actions trigger server request, server returns an HTML page to the client again after completion processing, Enables users don't have to look at the rotation of the cursor waiting for the server to be returned, so we can produce a good user experience.

\section{SYSTEM DESIGN}

\section{A. System Architecture}

The system uses the popular B/S three layer architecture, using ASP.NET and AJAX technologies; the backstage database is SQL Sever 2005, data layer using ADO.NET data access technology, to the operation of the database in the form of class encapsulation. With this architecture, server-side configuration requirements are lower. System deployment, application, upgrade and maintenance are convenient [6].

The user through the browser to access on the client, the client does not need to install additional applications, easy to use. Its biggest advantage is its security, user can only access the data layer by logic layer, reducing the entrance point, blocked many dangerous system function [5]. Otherwise, it can make full use of network computing functions, support distributed computing environment better.

\section{B. System Function Module Design}

The main functions of the system is divided into front and back two parts, at the system front-end is responsible for implementing the login related functions, mainly includes view interfaces of student login, new user registration, login, exam, exam questions, exam, scores query selection and test record check, test access and test related knowledge point and provides a series of validation function.

The system backend is responsible for implementing the function of teachers and administrators, mainly includes the system maintenance interfaces of the backstage login, user information maintenance, test question maintenance, teachers set test, the new test, performance management and other functions maintenance, and provides the functions of large data import, export and some query[7,8,9]. The system function module is shown as "Fig. 2".

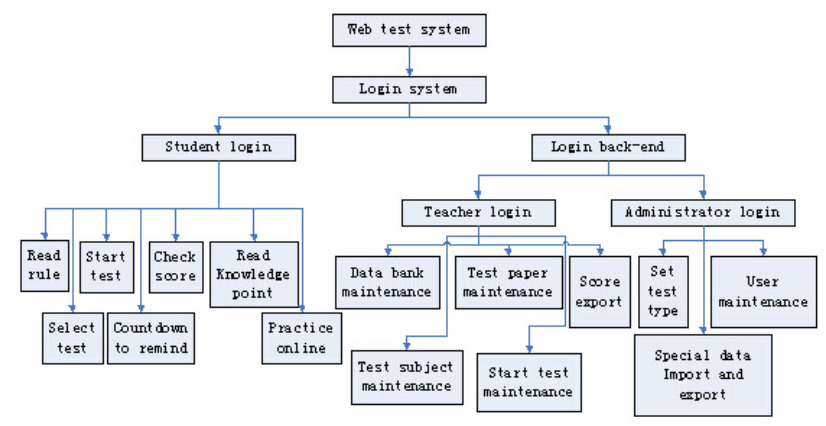

Figure 2. System function module.

Using the above module design, the system can effectively use the functional independence, realizes each module is an independent and a single small system, convenient in maintenance and extension in the late.

\section{System Process Design}

To highlight the application and convenient management, the System mainly includes the following three roles: teacher, student and system administrator. It 
mainly based on the user authentication to realize the role allocation.

1) teacher identity: When a user login as the teacher identity, can enrich the related curriculum item bank; can create test and release test, includes the test students, examination venues, test time, exam course, examination paper production and so on, also can be a key to generate the follow-up documents which like corresponding examination score, score analysis table.

2) Student identity: Student identity can read the release test information, read Test instructions, select the test subjects, take the exam, check the results after hand in an examination paper, online training, Check the history test and look over knowledge point.

3) Administrator identity: Administrator identity can maintain information on students and teachers.

The functional flow diagram is shown as "Fig. 3".

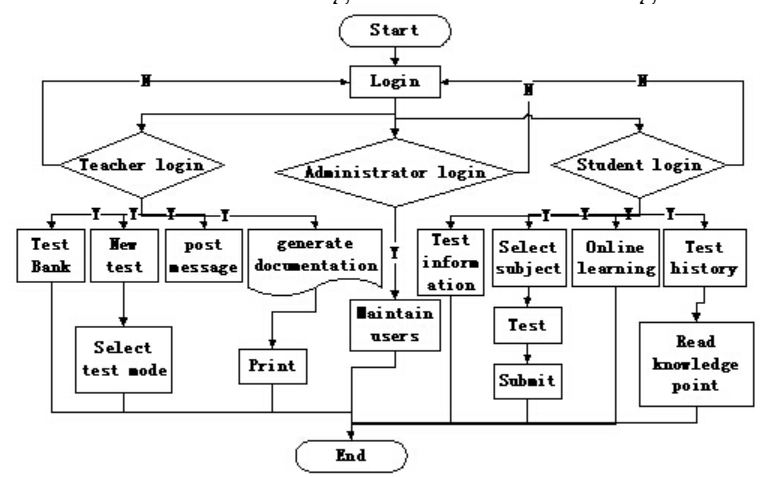

Figure 3. Functional flow diagram.

System provides special interfaces for each user, when a user name or password is not correct, the system page should be redirected to the login screen. The same, in every page, did the judgment on the legality of the user, when a user does not have the page operation permissions, the system page should be redirected to the login screen. In this way, it can effectively avoid the information leakage for the illegal users.

Using this process design, the system can effectively separate the each type of role process, has realized permissions separation of user the student, teacher and administrator, achieve the goal of the noninterference in each process

\section{Database Design}

Database design is extremely important in the system design process, a good database structure can reduce the redundancy, reduce system storage resources, improve storage efficiency, ensure the data integrity. The system database should design from system actual demand, and have a certain Prospective, keep the good scalability, Not because of a minor change in the future business, causing the structure of the system database is invalid.

Based on the system function analysis, the information requirements are as user of system, professional, course for student, students taking exams, the question of knowledge, test paper, Results table and Student paper , in order to eliminate redundancy, by modifying and reconstruction, the main entities and its properties are defined as shown in table 1 .
TABLE I. ENTITY AND PROPERTY

\begin{tabular}{|l|l|}
\hline \multicolumn{1}{|c|}{ Entity } & \multicolumn{1}{|c|}{ Properties } \\
\hline User & ID, name, password, permissions \\
\hline Professional & ID, name \\
\hline Course & ID, professional ID, name \\
\hline Student & ID, name, password, professional ID, class \\
\hline Test question & $\begin{array}{l}\text { ID, course ID, responsible person ID, contents, subject } \\
\text { type, various options, the standard answer, values, } \\
\text { knowledge point }\end{array}$ \\
\hline Test paper & $\begin{array}{l}\text { ID, course number, responsible person number, time, } \\
\text { place, test purpose, test mode }\end{array}$ \\
\hline Results table & record number, ID card number, test number, grade \\
\hline Student paper & $\begin{array}{l}\text { ID, test paper ID, Student ID, item number summary, } \\
\text { answers summary }\end{array}$ \\
\hline
\end{tabular}

The E-R diagram describes the conceptual data model of system; the E-R diagram of relationship between system entities is shown as "Fig.4".

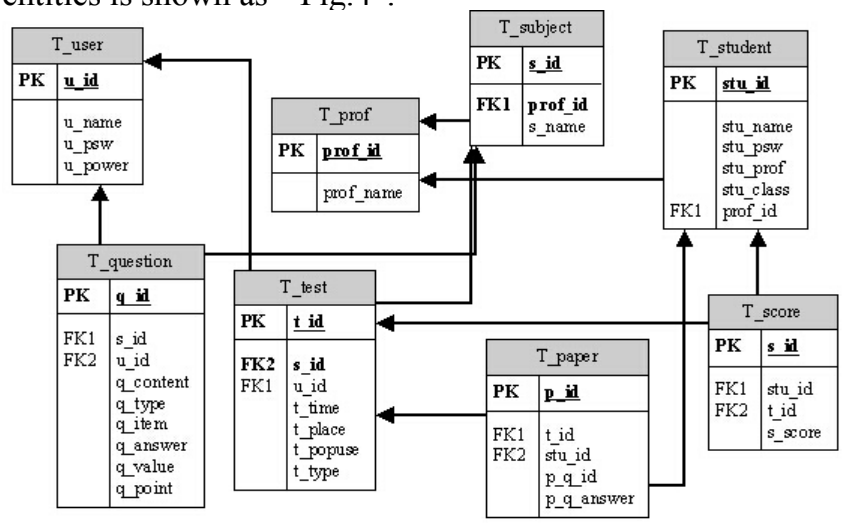

Figure 4. E-R diagram.

\section{SECURITY STRATEGY}

This system is a WEB application, so the system security is very important. Many aspects in the system design and implementation process to ensure the system safety, as follows:

1) Using authentication:Configure the application Forms authentication mode, and then the visitor access to the specified URL, submit the user name and password By submitting the page, Forms authentication system will be redirected to the specified login page while the request without authentication.

2) Using Authorization:Authorized by the application configuration file, or in the program to verify whether the user is entitled to visit the resources.

3) Using the verification code: The verification code can prevent malicious, garbage data submission.

4) Using stored procedures: Using stored procedures can prevent SQL injection, the type of system database operation uses stored procedure parameter and not string concatenation parameter.

5) Using low Privilege:The database connection account is given a low privilege, not an administrator privileges, try to use the lowest access account. 


\section{TEST AND ANALYSIS}

the web-based test system running environment is described as follows, on the web server, operating system selects Microsoft Windows Server 2008 R2 CHS with Configuring Internet information services, installs Microsoft Office2003 software; the database management system uses Microsoft sqlserver2005 which deploys to a separate computer or web server. The client side only needs any browser that can use the system.

The first tested is tried out with 200 participants on-line at the same time; we found that the concurrent problems connecting to the database, the other problem is session time out by setting the concurrent PARAM and session PARAM in the system configuration file, solve related problems.

During more than a year, the system has been applied in different fields; the running condition of the system is shown as Table 2.

TABLE II. THE RUNNING CONDITION OF THE SYSTEM

\begin{tabular}{|c|c|c|c|c|}
\hline Year & application area & people & computer & $\begin{array}{c}\text { Accuracy } \\
(\%)\end{array}$ \\
\hline 2012 & $\begin{array}{l}\text { new student Basic } \\
\text { computer proficiency }\end{array}$ & 2046 & 300 & 98 \\
\hline 2012 & $\begin{array}{l}\text { Basic computer course } \\
\text { examination }\end{array}$ & 935 & 150 & 100 \\
\hline 2013 & Single recruit exam & 145 & 300 & 100 \\
\hline 2013 & $\begin{array}{l}\text { new student Basic } \\
\text { computer proficiency } \\
\text { test }\end{array}$ & 3381 & 300 & 100 \\
\hline 2013 & $\begin{array}{l}\text { Provincial skills } \\
\text { contest }\end{array}$ & 108 & 300 & 100 \\
\hline 2013 & $\begin{array}{ll}\begin{array}{l}\text { College } \\
\text { competition }\end{array} & \text { skills } \\
\end{array}$ & 177 & 300 & 100 \\
\hline
\end{tabular}

As can be seen from Table 2, the performance of the system does not take place obvious change with the people number variation, but it affected by the types of the browser. To solve compatibility of different browsers standard, is a long-term research problems.

In the first time the formal application, it emerges a small number of errors, the reason is caused by improper data field length in the data table, The system revised version is into a stable performance, After many experiments with different scale and different fields, it proofs that this web application system's stability, concurrency and maintenance can achieve the expected purpose, have good application value.

\section{CONCLUSIONS AND FUTURE WORKS}

This paper researches a web-based test system, based on $\mathrm{B} / \mathrm{S}$ three layer architecture, designed a platform for different fields on-line test web application, studied on the related technologies, system architecture, process design, database conceptual design, security design, and by calling server-side winword.exe process, realized the paper making method in a variety of ways. The system has been applied in some field and had stable and reliable performance. The next work is going to improve operation platform of the system so that can support mobile devices, realize test in the mobile Internet.

\section{REFERENCES}

[1] Wang Xiaofeng, "Based on ASP.NET and AJAX intelligent network test system," Applied Mechanics and Materials, 19791982,2013.

[2] Liu-Dan,Ma- Shi-Xia ,and Guo-Zu-Hua, "Using ASP.NET and AJAX to build late-model community digital management system,” Int. Workshop Educ. Technol. Comput. Sci., ETCS ,vol.1, 2010, pp.555-558, March 2010.

[3] Wang, Huadong, "Research of key technologies in development in thesis management system," Advances in Intelligent and Soft Computing, v 128, p 317-322, November

[4] Wang, H,and Yang, J, "Research and application of web development based on ASP.NET 2.0+Ajax," ICIEA 2008, pp.857860, 2008.

[5] Glotzbach, Ronald J., Burton, Terry L., and Co, Betty, "Online instructor evaluations: A Web-based application",Journal of Industrial Technology,24(4),2008

[6] Sadoun, Balqies., Al-Bayari, Oma, and Al-Rawashdeh, Sami., "The development of a web mapping system and its application",ICE-B 2011 - Proceedings of the International Conference on e-Business ,pp.22-28,2011.

[7] Ya-Ning, Shi-Kai,and Shen, Wang, "Research and implementation of intelligent test paper for an exam system based on web.," 201 International Conference on E-Business and E-Government, ICEE2011 - Proceedings, p 8140-8142, 2011

[8] Marzic, S., Jugo, I.,and Radovan, M., "Developing dynamic web applications: "Exam scheduler"," MIPRO 2011 - 34th International Convention on Information and Communication Technology, Electronics and Microelectronics - Proceedings, pp.1127-1132, 2011

[9] Lister, Raymond, Jerram, Peter, "Design for Web-based ondemand multiple choice exams using XML," Proceedings - IEEE International Conference on Advanced Learning Technologies, ICALT 2001, pp. 383-384, 2001.

[10] Dalai, Asish Kumar, Jena, and Sanjay Kumar, "Evaluation of web application security risks and secure design patterns," ACM International Conference Proceeding Series, p 565-568, 2011 